\title{
Digital experience design of qinhuai lantern based on user journey map
}

\author{
Li nannan ${ }^{1 *}$, Lu zhangping ${ }^{1}$, Li mingzhu ${ }^{1}$, Ding ying \\ Department of Industrial Design Art School of Jiangsu University Zhenjiang, China
}

\begin{abstract}
This research extends the design concepts and research methods of user journey maps to the field of intangible cultural heritage, and selects the typical lotus lantern material package in Qinhuai lanterns as an example to explore the digital experience design of lanterns. On-site research methods are used to understand users, define target users and build personas. AEIOU recording framework is used to record user behavior, analyze and reorganize user behavior with card classification, and summarize user behavior flow. The Likert Smiley Scale is used to ask children to evaluate specific behaviors, get emotional curves, and summarize design opportunities from the perspective of valley filling. Given the pain points in the experience of the target user children and the indirect user parents, the lotus lantern material package is digitally redesigned from four perspectives of materials, connection methods between materials, functions and storage to meet the users' emotional experience. This research method and process have certain reference significance for experience design research on the same type of intangible cultural heritages.
\end{abstract}

\section{Introduction}

Qinhuai colored lanterns, also called Qinhuai lanterns, originated from the Six Dynasties and were well-known as "Qinhuai lanterns are the best in the world" in Ming Dynasty [1]. Qinhuai Lanterns are a special folk culture in Nanjing and Zhenjiang and integrate paper-folding, painting, calligraphy, paper-cutting, needlework, sculpture and other arts. The making of lanterns combines carpentry, painters, colored drawing, clay sculpture, knotting and other arts into one characteristic art skill and conveys profound cultural connotations. On the one hand, Qinhuai Lanterns harbor the yearning of people for a trouble-free, peaceful and happy life. On the other hand, Qinhuai Lanterns reflect to some extent the social life, customs and cultures of Nanjing and Zhenjiang in different eras, and thus are of huge values for studying the humanities and history of these two cities.

With the intensification of international cultural and economic competition and the expanding of modern industrial civilization, the development of colored lanterns has been restricted. As important carriers and messengers of intangible heritages, inheritors are inheriting and propagating colored lanterns by making and selling material packages. Material packages are the most direct and vivid way of inheritance, but are encountered with some problems. Firstly, material packages are designed and made by older inheritors, but the end users of material packages are mostly children. The existing material packages are made without studying the target users. Secondly, Qinhuai Lanterns used to be the leading role in life, but due to economic growth, the modern life has been changed tremendously. While the cultural connotations of lanterns are preserved, redesign is needed to make the lanterns meet the lifestyles and living needs of modern people.

\section{Research advances in Qinhuai lantern experience design}

Starting from the logic of "matter" and "thing" in an intangible culture-- lotus lanterns, Chen Jiajia explored the modularized structures of lotus lanterns based on service design [2]. Wang Qian et al. targeted at customized service design innovations and developed an online customization service platform for Qinhuai lantern cultural and creative products [3]. Li Juan et al. analyzed the artistic values and production process of coloured lanterns, and proposed the idea of APP-based improvement of traditional handicraft experience [4]. A new type of portable and reusable lanterns that can be easily assembled and dismantled was designed [5]. Liu Junzhe et al. combined reusable elastic materials with 3D printing connecting parts, and designed a type of spliced Qinhuai lanterns [6]. The Jinling creation team from Nanjing Arts Institute deconstructed traditional lanterns by using a modern design method, and redesigned souvenirs $\cdot$ Nanjing lotus lanterns by flattening the lanterns.

Many attempts on experience design of Qinhuai Lanterns have been made by the academic circle, but the existing studies are mostly based on literature searching. There is no user-based research on the experience design of lanterns from the theoretical perspective. In the experience economy era, users are increasingly seeking the emotional experience of products in addition to

*Corresponding author: 763106949@qq.com 
product functions. Creating perfect user experience relies on in-depth user research and user-centered design.

\section{Research methods}

\subsection{User journey maps}

User journey maps can visualize the behaviors, feelings, views and psychological activities (positive, negative and neutral) of users when using a specific product or enjoying a certain service. This approach helps designers in analyzing the contact points of the user in each step, discovering the pain points and happy points, extracting the opportunity points, and effectively improving the existing product or service [7]. A user journey map consists of personas and user journey. The personas are also called character portrays, and are the virtual user models that can express the demands, targets and characteristics of specific users and are created after interviewing and directly observing real users ${ }^{[8]}$. Personas help designers to visually feel the ultimate users [8]. The common dimensions of user journey include experience stage, behavior flow, user emotion, pain points, and opportunity points [9].

\subsection{On-site research method}

Since the cognitive ability and focusing ability of children are limited, the research method for children users is different from ordinary users. During on-site research, the investigators go to the real occurring place of an event and enter on-site, and sometimes have to be participants who will live together with the research subjects. On-site research is very suitable for studying children [10]. Onsite research integrates the authenticity of natural research and the strictness of experimental research, and is a new method that combines the two. Generally, on-site research requires the investigators to go to "field" and observe things under the natural state, or to create a "natural environment", in which they can study in a natural way.

\section{Construction of user journey map for Qinhuai lantern experience design}

\subsection{Target users of Qinhuai lantern experience design}

Montessori with years of experience in pre-school education holds that the 6- to 9-year-old period is a culture sensitive stage. Together with the age distribution characteristics of Chinese children, we targeted at children going to the first or second grade of elementary schools, and these children are mostly 7 to 9 years old. The classification of children helps to understand the characteristics of target users, and we conducted our research on this basis. When we sent out recruitment orders for target users, our research was supported by teachers, children and parents from Zhenjiang Tomato Field Arts Center. Finally, 9 children were involved, including 3 boys and 6 girls.

\subsection{Construction of personas}

The primary task of user journey maps is the construction of personas. The personas of Qinhuai Lantern experience design were created by three stages.

In the first stage of culture experience and user familiarization, users were observed by organizing children to participate in Qinhuai Lantern cultural experience activities (Fig. 1). Cultural experience activities were conducted in the Tomato Field Arts Center, which was a relatively natural environment for these children. During this process, the investigators selfpositioned as peers of children and were fully involved in the lantern making activities of children. We shared their laughter, anger, sorrow and happiness, and built confident, friendly and harmonious relations with them, which will underlie the subsequent communications with these children. The cultural experience lessons consisted of three parts: (1) icebreaking meeting: self-introduction by all people; (2) introduction of Qinhuai Lanterns cultures on PPT; (3) provision of rabbit lantern material packages, and making rabbit lanterns with the children. The activities were recorded by videos and audios, and the behaviors of children were observed on videos, including the familiarity with the material package, the proficiency of hand making, and the attitudes towards the material packages. According to the complete scenario veins of children making rabbit lanterns, the target users were divided into proficient users, intermediate users, and new users.

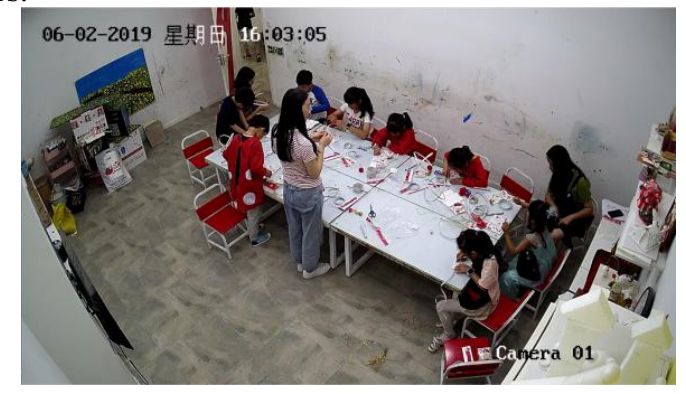

Figure 1. Participation of children in Qinhuai Lanterns cultural experience

The second stage was interviewing and definite classification. Parents, as the most intimate users of children, best know the characteristics of their own children. We deeply discussed with parents one-to-one about the life habits of their children, especially the attitudes toward the manual art experience activities (Fig. 2). After the interviewing, we found the proficient users loved to experience all kinds of hand-making activities, and they asked to take part in hand-making activities at least twice every week. They were familiar with most of the experience materials, and severely demanded for manual experience activities. The intermediate users nonperiodically experienced hand-making activities under the company of their parents, and they were familiar with ordinary hand-making stuff, but they did not think handmaking activities were necessary for them every week. The new users did not initiatively join in these hand- 
making activities, and will only take part when such activities were organized in their schools. They were unfamiliar with the materials, and can finish the experience activities only under hand-in-hand guidance.

The third stage was determination of target users and plotting of user maps. According to the first two stages, the proficient users are those who are extremely fond of hand-making experience, and they have rich experience and strong demands. The intermediate users and new users have fewer demands for hand-making experience. Hence, the proficient users were selected as the first batch of target users. By combining on-site research and parent interviewing, we plotted the user maps of the proficient users, including living conditions and time in handmaking experience, and created simple scenes (Fig. 3).

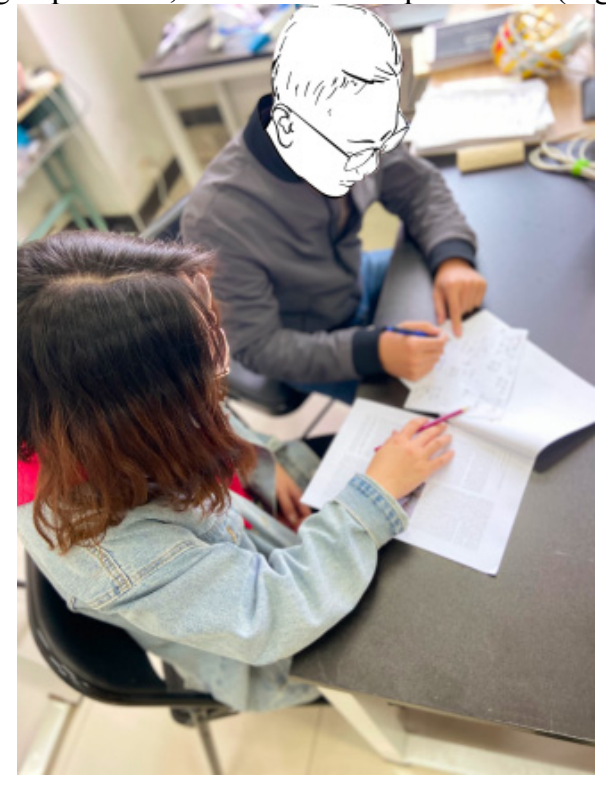

Figure 2. Deep interviewing with indirect users

Basic information
Name: Xi'er
Status: proficient user
Age: 8 years
Hobbies: painting, hand works
Disposition: active, naughty, lovely
Joined training classes: English, arts, dance, calligraphy
Living status: She lives with parents. Her parents are both teachers and busy, but
they take part in weekend parents-kids activities of art classes. Her parents often
preserve the final works of her handwork experience activities to commemorate the
growing of Xi'er. However, some manufactured works are unstorable and will be
discarded due to deformation after long time.
Time of experience: At weekends or the leisure time after classes every afternoon,
Xi'er loves to manually work with the materials her father has bought, including
paper folding, clay diy, and knitting. She mainly makes by reading manuals or
watching videos, and her father occasionally companies her at weekends. Xi'er is
very satisfied with the works she has independently finished.
Scene construction: Today is Sunday. Xi'er went to English training class in the
moming, and took out a material package after lunch with her parents. She planned
to independently finish it during the noon nap of her parents and expected to surprise
them. She also wants to share the finished lantern with her good friend Lili.

Figure 3. Role model of the proficient users

\subsection{Plotting of user journey maps}

We provided the users Lotus lantern material packages, experienced lotus lantern making together with the proficient users through on-site research, and videorecorded their gestures, expressions and other nonverbal interactions during this process.

Firstly, their behaviors were deeply explored by video playback, and their behavioral process of lotus lantern making was recorded by using AEIOU organization frame [11] (Fig. 4). From the AEIOU frame, 23 user contact points were extracted, and recorded on cards. These contact points were analyzed and reorganized together with the proficient users. The lotus lantern making tasks were decomposed into a series of immediately connected user behaviors, and 3 experience stages were summarized, including preparing, producing, and ending. These 23 contact points were classified into first-level behaviors and second-level behaviors.

Secondly, to attract the children, help them understand and make the grading more effective, we adopted the Likert Smiley Scale [12] to ask the children to grade each contact point of the two levels of behaviors from three dimensions (experience, fun, difficulty) (Fig. 5). Together with the performances and grades of the children during lotus lantern making, we objectively plotted user emotion curves. The user experience maps show that user experience can be improved by elevating peaks, filling valleys, optimizing the key time point of user contact, "wind head", "leopard tail", extending customer experience journey, jumping over stages and activities, reranking of service stage and activities, or thorough redesign [13]. In this study, user experience was improved mainly by filling valleys, which was aimed to make users feel less pain at the pain point. From the user emotion curves, we know the low-spirit points of users below the emotion horizontal line include: D hands hurt by clips during frame building; E1 glues sticking hands during petal pasting; E2 damage of transparent strips; G cannot find the small splicing holes; I cannot knot when fastening the rod; $\mathrm{N}$ petals falling off during lantern playing. The low-spirit points of the proficient users on the emotion curves, or namely the pain points of target users during lotus lantern experience, were summarized, and the potential opportunity points were analyzed (Fig. 6), which will guide the direction in the subsequent design. 


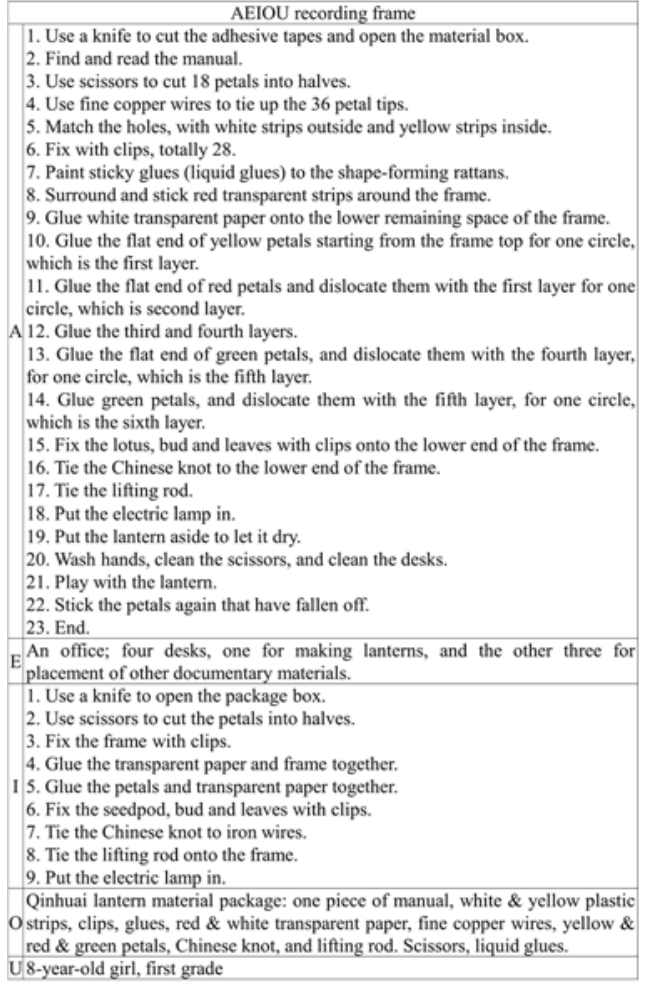

Figure 4. AEIOU recording frame of proficient users

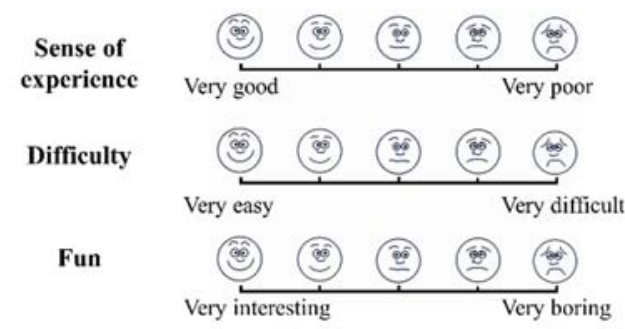

Figure 5. Likert Smiley Scale

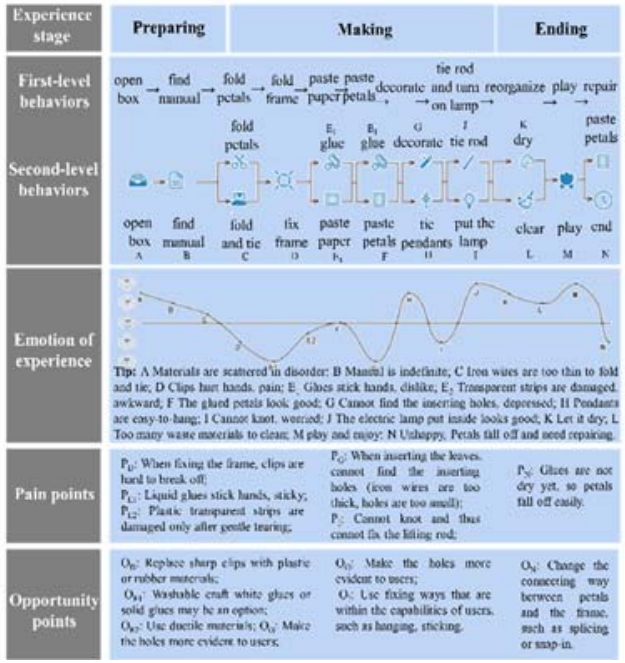

Figure 6. Journey maps of proficient users

\subsection{Determination of opportunity point for Qinhuai Lantern digital experience design}

Parents as indirect users play important roles and actions in the life of children, so research on children must involve the attitudes of parents. Through depth interviewing, we knew the attitudes of parents towards Qinhuai lotus lantern handwork material packages, as the parents all regarded Qinhuai Lanterns as traditional cultures, and affirmed the manufacturing of Qinhuai Lanterns and understanding of cultural connotations by children. However, the lantern material packages still have three limitations. Firstly, the final lanterns are too large in volume and are unsuitable for carrying and placement. Secondly, the material packages are disposable products and basically cannot be utilized in modern life. Thirdly, the final lanterns are undismountable and unstorable and thus will be discarded after certain time of placement.

Together with the journey maps of target users (children) and depth interviews with indirect users (parents), we summarized the pain points encountered during experience of lotus lanterns material packages, and analyzed the causes for the problems faced during handmaking and extracted the design opportunity points (Fig. 7). Since storable products can be conveniently carried, so we suggest to improve user experience from four aspects, including materials, connecting way between materials, functions, and storage.

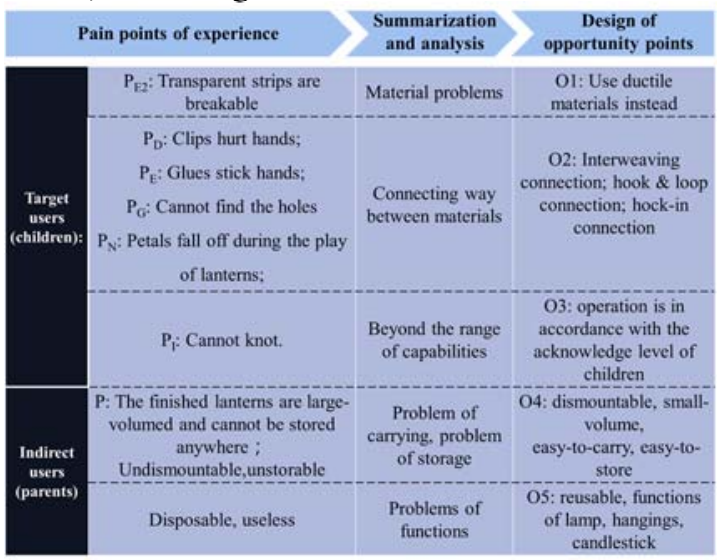

Figure 7. Analysis of opportunity point for Qinhuai Lantern experience design

\section{Practice of Qinhuai Lantern digital experience design}

According to comprehensive analysis, the computer digitization technology is used to design and simulate the digital experience of the new lotus lanterns. The material package involved mainly includes 1 circular rings, 2 hanging ropes, 3 petals, 4 seedpod, 5 pushing switch, 21 center hole, 4a seedpod cover, 41 circular holes, 42 rectangular holes, 5a switch outer-wall bulges, 51 stretching pillars, 51a springs, 6 stretching pillar controlling parts, 61 bulges of stretching pillar controlling parts, 7 external column, 71 external column bulge (1), 72 external column bulge (2) (Fig. 8).

The digitally redesigned lotus lanterns materials were food-grade soft silica gels, which are safe and ductile and solve the materials-induced pain point $\mathrm{P}_{\mathrm{E} 2}$. The materials were mainly assembled by spicing, which removed redundant assemble parts and solved the pain points $P_{D}, P_{E}$ 
and $\mathrm{P}_{\mathrm{G}}$ caused by between-material connecting way. The lower projecting part of lotus petals was wedged into the rectangular hole on the seedpod and thus did not easily fall off, which solved pain point PN. The new lotus lanterns material package was modularized and can be easily assembled, reinstalled, stored and carried, which solved the pain points of indirect users (parents), including "cannot be placed, dismantled and stored". Traditional lotus lanterns are usually hung on trees to express the idea of "country in peace and people in safety". People long for better life and hang lotus lanterns in their homes to express the ideas of happy and peaceful families. In this design, we inherited the semantics of "hanging" lotus lanterns, and through the connection by circular rings and hanging ropes, we created the current popular hanging things, which solved the pain point of "disposable products, uselessness" faced by traditional festive lanterns. The new type of lotus lanterns (Fig. 9) was manufactured by 3D printing for verification. The new festive lanterns inherit the crafts, shapes and implications of traditional lotus lanterns, and solve the pain points of traditional lotus lanterns material packages during user experience. Their elegant shapes accord with modern lifestyle, which brings this intangible cultural heritage to life again.

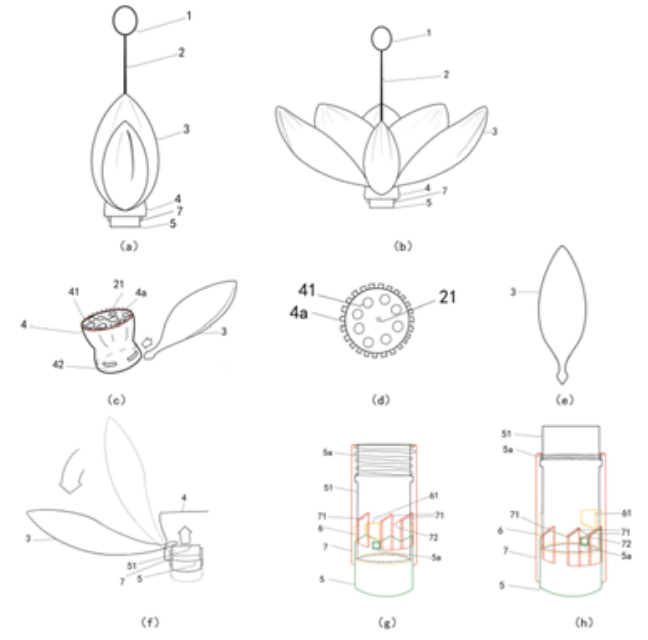

Figure 8. Experience materials of Qinhuai Lantern - lotus lanterns

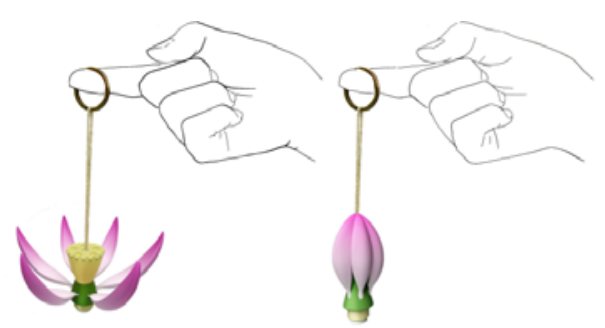

Figure 9. Final plan of Qinhuai Lantern experience design

\section{Conclusions}

The typical lotus lanterns material package of Qinhuai Lanterns was studied, and the digital experience design of Qinhuai Lanterns was explored using the concepts and methods of user journey maps. Through on-site research, the children users were placed in a "natural environment" to observe their real states, and target user journey maps were plotted through objective and strict analyses. By combining user journey maps and depth interviews with indirect users (parents), we summarized the pain points faced during manufacturing and experiencing of the existing Qinhuai lotus lantern material package and extracted opportunity points. Then the Qinhuai lotus lantern material package was digitally redesigned, and the experience of user manufacturing was improved. The research methods and ideas adopted in this paper are relatively strict for the digital experience design and analysis of Qinhuai Lanterns, which can be meaningfully referred to by the experience design and research of other intangible cultures of the same type.

\section{References}

1. Xu Yang. Study on Civil Festival Lantern Art in Qinhuai Area Affected by Patriarchal Clan System. Journal of Jinling Institute of Technology: Social Science Edition, 2011, 25(3): 59-63.

2. Chen Jiajia. Redesign of Immaterial Culture Heritage of Lotus Lantern Based on Service Design Thinking. Hundred Schools In Art, 2018, 34(03): 235-239.

3. Wang Qian, Liu Junzhe, Liu Yan. Service Design Of Qinhuai Lantern Customization Cultural And Creative Product In"internet+"era. Design, 2019, 32(01):16-19.

4. Li Juan, Liu Junzhe, Shao Anning. Conceptions on Cultural Inheritance and Informatization Conservation of Traditional Nanjing Qinhuai Lanterns. Family of the Drama, 2019, 302(02): 235236.

5. Patent of utility model, CN 07906909 U, Jinling Institute of Technology.

6. Liu Junzhe, Zang Ke. China College and University Design Work Collection Yearbook, 2016.

7. Yang Lili. Optimized Design of Medical Care Preregistration Systems based on Theory of Service Design. Southeast University

8. Steve Mulder, Zivv Yarr. The User Is Always Right: A Practical Guide to Creating and Using Personas for the Web. Beijing: China Machine Press, 2007.

9. Bella Martin, Bruce Hanington. Universal Methods of Design. 2013.

10. Lu Chang'e, Wang Yong. On the spot study method and its value in pre- school education study--See from education ecology. Journal of China Women's College(Social Sciences Edition), 2004, 16(5):59-62.

11. Hu Fei, Du Chenteng. Comparative Study on User Observation Frames. Journal of Nanjing Arts Institute Fine Arts \& Design, 2012(2): 178-184.

12. Read, J.C., et al. An Investigation of Participatory Design with Children - Informant, Balanced and Facilitated Design. Procs Interaction Design and Children,2002,53 - 64.

13. Li Sida, Ding Zhaochen. Introduction into Service Design. Beijing: Tsinghua University Press, 2018. 